\title{
ON THE HYPERBOLIC DISTANCE OF $n$-TIMES PUNCTURED SPHERES
}

\author{
TOSHIYUKI SUGAWA, MATTI VUORINEN, AND TANRAN ZHANG
}

\begin{abstract}
The length of the shortest closed geodesic in a hyperbolic surface $X$ is called the systole of $X$. When $X$ is an $n$-times punctured sphere $\widehat{\mathbb{C}} \backslash A$ where $A \subset \widehat{\mathbb{C}}$ is a finite set of cardinality $n \geq 4$, we define a quantity $Q(A)$ in terms of cross ratios of quadruples in $A$ so that $Q(A)$ is quantitatively comparable with the systole of $X$. We next propose a method to construct a distance function $d_{X}$ on a punctured sphere $X$ which is Lipschitz equivalent to the hyperbolic distance $h_{X}$ on $X$. In particular, when the construction is based on a modified quasihyperbolic metric, $d_{X}$ is Lipschitz equivalent to $h_{X}$ with Lipschitz constant depending only on $Q(A)$.
\end{abstract}

\section{INTRODUCTION}

One of the fundamental research themes of geometric function theory is the study of the way in which distances between points are transformed or distorted under analytic or meromorphic functions $f: \Omega_{1} \rightarrow \Omega_{2}$, where $\Omega_{1}$ and $\Omega_{2}$ are subdomains of the Riemann sphere $\widehat{\mathbb{C}}=\mathbb{C} \cup\{\infty\}$. For this purpose distances are measured in terms of metrics. Several metrics are commonly used: the euclidean, chordal and hyperbolic metrics are some examples. The numeric value of the hyperbolic distance $h_{\Omega}(z, w)$ of two points $z, w$ in a plane domain $\Omega \subset \mathbb{C}$ with $\operatorname{card}(\widehat{\mathbb{C}} \backslash \Omega) \geq 3$ does not merely reflect "the distance between points" but also "the location of the points with respect to boundary" and "the metric structure of the boundary". These facts, along with its conformal invariance, make the hyperbolic metric a powerful tool in geometric function theory 3 . Often it is more natural to use the hyperbolic distance $h_{\Omega}$ than to use the euclidean or chordal metric. The study of the hyperbolic metric is a part of the wider context where metrics and their applications to geometric function theory have a key role. For instance, in the study of quasiconformal maps of subdomains of $\mathbb{R}^{n}, n \geq 2$, several new metrics have been introduced during the past few decades, with the purpose of generalizing hyperbolic metric to subdomains of $\mathbb{R}^{n}, n \geq 3$. One of these "hyperbolic-type" metrics is the quasihyperbolic metric, which has become a popular tool applied by many authors [12], [16], [37] etc.

The hyperbolic metric of a general plane domain $\Omega \subset \mathbb{C}$ with $\operatorname{card}(\widehat{\mathbb{C}} \backslash \Omega) \geq 3$ is defined in three steps. First, in the case of the unit disk $\Omega=\mathbb{D}$, one can give an explicit formula for the hyperbolic distance [6]. Second, for a simply connected domain, the definition reduces to the case of the unit disk by use of the Riemann mapping theorem. Third, in the case of a general domain $\Omega \subset \mathbb{C}$, the hyperbolic metric is defined as a Riemannian metric with

File: svz20170719.tex, printed: 2018-10-20, 13.54

2010 Mathematics Subject Classification. Primary 30C35; Secondary 30C55.

Key words and phrases. hyperbolic metric, systole, Voronoi diagram.

The authors were supported in part by JSPS Grant-in-Aid for Scientific Research (B) 22340025. 
the density function $\lambda_{\Omega}=\lambda_{\Omega}(z)|d z|$ given in terms of the universal covering mapping (see below or [20]). Distances between points are then obtained by minimising the line integrals over the paths, the hyperbolic lengths of the paths, connecting the points. The reader should notice that in the literature, the word "metric" refers often to the density function of the Riemannian metric rather than to the distance function "metric" in the sense of topology.

Of these three cases, the extremal cases are the first case, when the domain $\Omega$ is the unit disk $\mathbb{D}$, and the third case, when the domain is the trice punctured sphere $\Omega=\mathbb{C}_{0,1}=\mathbb{C} \backslash\{0,1\}$, or a domain conformally equivalent to one of these. The unit disk case is well-known [6] and the density of the metric is $1 /\left(1-|z|^{2}\right)$. The case of $\mathbb{C}_{0,1}$ is much more difficult and leads to classical special functions such as elliptic functions and their representation as infinite products, see [2, 17, 20], and the density $\lambda(z)=\lambda_{\mathbb{C}_{0,1}}(z)$ of the metric satisfies

$$
\lambda(z)=\frac{1}{2|z||\log | z||}+O(1) \quad \text { as } z \rightarrow 0, \quad \lambda(-1)=\frac{2 \pi^{2}}{\Gamma(1 / 4)^{4}} \approx 0.114237 .
$$

Functional identities for classical special functions also yield identities and numerical approximations for $\lambda(z)$ [30]. The case of a general domain lies between these extreme cases which correspond to steps one and three of the above definition. In the case of the unit disk, we have not only an explicit distance formula, but also a rich structure of the hyperbolic geometry which includes counterparts of some results of the euclidean geometry [6]. In the case of a general simply connected domain, we loose all this because the Riemann mapping function is not explicitly known, but what remains are good upper and lower bounds for the density [12, (3.2.1)], and if the domain is not only simply connected but also uniform, then the hyperbolic distance has upper and lower bounds in terms of the distance ratio metric or the $j$-distance, [12]. In the case of a general plane domain neither good upper nor lower bounds for the hyperbolic distance exist, only there are upper and lower bounds for the density function of the metric. These bounds depend on the geometry of the domain and on the metric structure of its boundary. Several particular cases, such as rectangles, convex domains, quasidisks, domains with isolated boundary points have been studied in the literature and the influence of thickness properties of the boundary such as uniform perfectness on the hyperbolic metric have been analysed [7, 8, 9, 19, 24, 31], [20, pp. 245-257]. In very few cases, the density function of the hyperbolic metric is known (annulus, punctured disk, strip domain [20, pp. 133-135]).

Many authors have studied these topics which bring together extremal problems of geometric function theory, classical hyperbolic geometry, special classes of domains, metric structure conditions of sets, and special functions [3, 2, 9, 10, 11, 17, 15, 18, 19, 21, 22, 23, 27, 30, 35. Mostly, the density function of the distance is studied. In spite of all this research, finding information about the hyperbolic distance $h_{\Omega}\left(z_{1}, z_{2}\right)$ induced by $\lambda_{\Omega}(z)|d z|$ in a general domain $\Omega$ seems to be very difficult. If good explicit bounds were known, one could for instance find new distortion theorems for analytic functions which are contraction mappings between hyperbolic spaces. For instance, Landau's and Schottky's theorems for analytic functions are closely connected with such estimates [15, pp. 707-708], [13], [17], [4, p. 210]. S. Rickman [28] proved a counterpart of Schottky's theorem for quasiregular mappings $f: \mathbb{B}^{n} \rightarrow \mathbb{R}^{n} \backslash A, n \geq 2$, where $A \subset \mathbb{R}^{n}$ is a finite set 
containing sufficiently many points and where $\mathbb{B}^{n}$ is the unit ball of $\mathbb{R}^{n}$. For that purpose he constructed a metric, which for $n=2$ is similar to the hyperbolic metric.

In the present paper, we will concentrate on the particular case when $\Omega$ is a punctured sphere of the form $X=\widehat{\mathbb{C}} \backslash A$ for a finite subset $A$ of $\widehat{\mathbb{C}}$ with $n=\operatorname{card}(A) \geq 3$. The aim of this paper is to introduce a comparison function, a new metric (in the sense of topology) $d_{X}$, whose values can be easily computed, with comparison bounds explicitly controlled by the geometry of $X$ or, equivalently, of $A$. It is not difficult to observe that the bounds cannot be taken uniformly when $n \geq 4$. Therefore, we have to pay attention to hyperbolic geometry of $X$ in an appropriate way. To measure "thickness" of $X$, it is customary to consider the systole sys $(X)$ of $X$, which is defined to be the minimal length of closed hyperbolic geodesics in $X$. For instance, it is known that $X$ tends to the boundary in the moduli space $\mathcal{M}_{0, n}$ precisely when sys $(X) \rightarrow 0$. However, by the reason described above, it is not easy to compute the systole.

In Section 2 we will introduce a quantity $Q(A)$ which can be computed by means of the cross ratios of quadruples of points in $A$ in a certain way, see (2.5). The main theorem in this section is Theorem 2.2 which roughly states that $Q(A)$ behaves like $\pi^{2} /$ sys $(X)$ when sys $(X)$ is small. We believe that this connection might be helpful in other aspects.

During the past few decades, several kinds of hyperbolic-type distances have been proposed such as the quasihyperbolic distance. However, near the punctures their behaviour is usually very different from that of the hyperbolic distance. In Section 3, we give a general method to construct a new distance $d_{X}$ from such a hyperbolic-type distance, say $\mu$, in such a way that the resulting distance is Lipschitz equivalent to the hyperbolic distance. We also give a concrete bound for these Lipschitz constants in Theorem 3.4. This construction was first proposed in [36] when $\mu$ is the euclidean distance.

Section 4 is devoted to some applications of this theorem. As one can easily guess, the euclidean distance is not suitable to construct a nearly hyperbolic distance. Typical hyperbolic-type distances are the so-called $j$-distance $(j$-metric) and the quasihyperbolic distance. However, these metrics do not satisfy the assumptions of Theorem 3.4. Thus, we will modify the construction of those metrics and obtain Lipschitz equivalent metrics which satisfy those assumptions. In particular, the resulting distance based on the modified quasihyperbolic distance is Lipschitz equivalent to the hyperbolic distance with bounds depending only on $Q(A)$ (see Theorem 4.4 below).

\section{Hyperbolic Geometry and CROSS Ratio}

We introduce here some basic notation and terminology. We denote by $\mathbb{D}(a, r)$ the open disk $|z-a|<r$ in the complex plane $\mathbb{C}$ for $a \in \mathbb{C}$ and $r>0$ and by $\overline{\mathbb{D}}(a, r)$ its closure, namely, the closed disk $|z-a| \leq r$. Also, let $\mathbb{D}^{*}(a, r)=\mathbb{D}(a, r) \backslash\{a\}$ and $\overline{\mathbb{D}} *(a, r)=\overline{\mathbb{D}}(a, r) \backslash\{a\}$.

Let $\Omega \subset \mathbb{C}$ be a domain with $\operatorname{card}(\widehat{\mathbb{C}} \backslash \Omega) \geq 3$ and with the hyperbolic metric $\lambda_{\Omega}=$ $\lambda_{\Omega}(z)|d z|$ of constant Gaussian curvature -4 . The hyperbolic length of a piecewise smooth curve $\gamma$ in $\Omega$ is defined to be

$$
\ell_{\Omega}(\gamma)=\int_{\gamma} \lambda_{\Omega}(z)|d z|
$$


We denote by $h_{\Omega}(z, w)$ the hyperbolic distance on $\Omega$ induced by $\lambda_{\Omega}$ and defined for $z, w \in \Omega$ by

$$
h_{\Omega}(z, w)=\inf \ell_{\Omega}(\gamma)
$$

where the infimum is taken over all curves joining $z$ and $w$ in $\Omega$. It is known that the infimum is attained by a hyperbolic geodesic. Here, a curve $\gamma$ is called a hyperbolic geodesic if its hyperbolic length is minimal among the homotopy class of $\gamma$ keeping the endpoints fixed. R. Nevanlinna's principle of the hyperbolic metric [26, p. 49], [15, p. 683] implies that if $\Omega_{1}, \Omega_{2}$ are domains in $\mathbb{C}$ with at least two finite boundary points and $f: \Omega_{1} \rightarrow \Omega_{2}$ is an analytic function, then

$$
h_{\Omega_{2}}(f(z), f(w)) \leq h_{\Omega_{1}}(z, w)
$$

for $z, w \in \Omega_{1}$, i.e. analytic functions are contractions in hyperbolic metric. This contraction property may be regarded as a very general form of the Schwarz lemma, which is the special case $\Omega_{1}=\Omega_{2}=\mathbb{D}$. Applying this result to the inclusion mapping we see that if $\Omega_{1} \subset \Omega_{2}$ and $z, w \in \Omega_{1}$, then

$$
h_{\Omega_{1}}(z, w) \geq h_{\Omega_{2}}(z, w) .
$$

Hence the hyperbolic metric is monotone with respect to the domain inclusion.

As pointed out in the introduction, the density $\lambda_{\Omega}(z)$ of the hyperbolic metric is not known for general plane domains $\Omega \subset \mathbb{C}$. Sometimes it is useful to study another metric with an explicit density function and use it as a comparison function for the hyperbolic metric. For such a purpose we introduce the quasihyperbolic distance $q_{\Omega}\left(z_{1}, z_{2}\right)$. The quasihyperbolic distance is defined for $\Omega \subset \mathbb{C}$ in the same way as the hyperbolic metric through the length minimizing properties of curves, but by replacing the hyperbolic density by $1 / \delta_{\Omega}(z)$, where

$$
\delta_{\Omega}(z)=\min _{a \in \partial \Omega}|z-a| .
$$

In the particular case when $\Omega=\mathbb{C}^{*}=\mathbb{C} \backslash\{0\}$, Martin and Osgood [25] gave an explicit formula of $q\left(z_{1}, z_{2}\right)=q_{\mathbb{C}^{*}}\left(z_{1}, z_{2}\right)$ as follows:

$$
q\left(z_{1}, z_{2}\right)=\sqrt{\left(\log \left|z_{1}\right|-\log \left|z_{2}\right|\right)^{2}+\left(2 \arcsin \frac{1}{2}\left|\frac{z_{1}}{\left|z_{1}\right|}-\frac{z_{2}}{\left|z_{2}\right|}\right|\right)^{2}}, \quad z_{1}, z_{2} \in \mathbb{C}^{*} .
$$

Note that, if the origin is an isolated boundary point of a hyperbolic domain $\Omega_{1}$, then for a fixed point $z_{0} \in \Omega_{1}, q_{\Omega_{1}}\left(z, z_{0}\right)=\log (1 /|z|)+O(1)$ as $z \rightarrow 0$ whereas $h_{\Omega_{1}}\left(z, z_{0}\right)=$ $\frac{1}{2} \log (\log (1 /|z|))+O(1)$ as $z \rightarrow 0$ (see (1.1) $)$. We also note that by (2.1) $q\left(t z_{1}, t z_{2}\right)=$ $q\left(z_{1}, z_{2}\right)$ for $t \in \mathbb{C}^{*}$.

It is well-known that an $n$-times punctured sphere $X=\widehat{\mathbb{C}} \backslash A$, where $A=\left\{a_{1}, \ldots, a_{n}\right\}$, is hyperbolic if and only if $n \geq 3$. In this paper, we will mainly consider hyperbolic punctured spheres, in other words, the $n$-times punctured sphere $X=\widehat{\mathbb{C}} \backslash A$ with $3 \leq$ $n=\operatorname{card}(A)<+\infty$. For convenience, we normalise $X$ so that $0,1, \infty \in A$ in the rest of the paper. In particular, $X \subset \mathbb{C}$.

We want to have a useful quantity which can be computed easily by the set of punctures $A$ of $X$. First let

$$
\widehat{Q}(A)=\max _{a_{j} \in A} \log ^{+}\left|\operatorname{cr}\left(a_{1}, a_{2}, a_{3}, a_{4}\right)\right|
$$


for a finite subset $A$ of $\widehat{\mathbb{C}}$ with $\operatorname{card}(A) \geq 4$, where $\log ^{+} x=\max \{\log x, 0\}$, the maximum is taken over all the quadruples of distinct points in $A$ and $\operatorname{cr}\left(a_{1}, a_{2}, a_{3}, a_{4}\right)$ stands for the cross ratio; that is,

$$
\operatorname{cr}\left(a_{1}, a_{2}, a_{3}, a_{4}\right)=\frac{\left(a_{1}-a_{3}\right)\left(a_{2}-a_{4}\right)}{\left(a_{1}-a_{2}\right)\left(a_{3}-a_{4}\right)}
$$

if none of $a_{j}$ is $\infty$, and otherwise it is defined as a suitable limit. Note the identities

$$
\operatorname{cr}\left(a_{1}, a_{2}, a_{3}, a_{4}\right)=\operatorname{cr}\left(a_{2}, a_{1}, a_{4}, a_{3}\right)=\operatorname{cr}\left(a_{3}, a_{4}, a_{1}, a_{2}\right)=\operatorname{cr}\left(a_{4}, a_{3}, a_{2}, a_{1}\right)
$$

and

$$
\operatorname{cr}\left(a_{2}, a_{1}, a_{3}, a_{4}\right)=1-\operatorname{cr}\left(a_{1}, a_{2}, a_{3}, a_{4}\right) .
$$

We set $\widehat{Q}(A)=0$ when $\operatorname{card}(A)<4$. Let sys $(X)$ be the infimum of the lengths of closed hyperbolic geodesics in $X$. This quantity is known as the systole of the surface $X$ and it gives a convenient way to measure "thickness" of the surface $X$ (see [32, 33]). Note that sys $(X)>0$ for the $n$-times punctured sphere, $n \geq 3$. Schmutz [29, Theorem 14] (see also [1, Theorem 7.2]) showed that

$$
\operatorname{sys}(X) \leq 2 \operatorname{arccosh}(3-6 / n)<2 \operatorname{arccosh} 3=4 \log (1+\sqrt{2})
$$

for an $n$-times punctured sphere $X$ with $n \geq 4$. We note that sys $(X)$ is not comparable with $\widehat{Q}(A)$ in a uniform way (see Example 2.6 given below).

We thus consider a modified quantity as described in the following. A pair of subsets $A_{1}, A_{2}$ of $A$ will be called an admissible partition of $A$ if each of $A_{1}$ and $A_{2}$ contains at least two points and if $A$ is the disjoint union of $A_{1}$ and $A_{2}$. We write $A_{1} \sqcup A_{2}=A$ for it. For an admissible partition $A=A_{1} \sqcup A_{2}$, we put

$$
P\left(A_{1}, A_{2}\right)=\min _{a_{1}, a_{1}^{\prime} \in A_{1}, a_{2}, a_{2}^{\prime} \in A_{2}} \log ^{+}\left|\operatorname{cr}\left(a_{1}, a_{1}^{\prime}, a_{2}, a_{2}^{\prime}\right)\right| .
$$

Note that $P\left(A_{1}, A_{2}\right)=P\left(A_{2}, A_{1}\right)$ thanks to (2.2) . Finally, we set

$$
Q(A)=\max _{A_{1} \sqcup A_{2}=A} P\left(A_{1}, A_{2}\right)
$$

for $A \subset \widehat{\mathbb{C}}$ with $4 \leq \operatorname{card}(A)<\infty$, where the maximum is taken over all the admissible partitions of $A$. Again, we set $Q(A)=0$ if $A$ consists of at most three points. Obviously,

$$
0 \leq Q(A) \leq \widehat{Q}(A)
$$

Note that both $Q(A)$ and $\widehat{Q}(A)$ are invariant under Möbius transformations.

A doubly connected domain $R$ in $\widehat{\mathbb{C}}$ is called an annulus. An annulus whose boundary consists of two circles (including lines) is called a circular annulus. Furthermore, if $R$ is bounded in $\mathbb{C}$ and if $\partial R$ consists of two concentric circles (centered at $a$ ), it is called a round annulus (centered at $a$ ). We recall that the modulus $\bmod R$ of an annulus $R$ is defined to be $\log \left(r_{2} / r_{1}\right)$ when $R$ is conformally equivalent to the round annulus $\left\{z \in \mathbb{C}: r_{1}<|z|<r_{2}\right\}$. We say that an annulus $R$ separates a set $A_{1}$ from a set $A_{2}$ if $A_{1}$ and $A_{2}$ are contained in different connected components of $\widehat{\mathbb{C}} \backslash R$. We can understand the geometric meaning of the quantity $P\left(A_{1}, A_{2}\right)$ in terms of separating circular annuli as follows. 
Lemma 2.1. Let $A_{1}$ and $A_{2}$ be disjoint finite subsets of $\widehat{\mathbb{C}}$ with card $A_{j} \geq 2$ for $j=1,2$. If $P\left(A_{1}, A_{2}\right)>0$, there exists a circular annulus $R$ separating $A_{1}$ from $A_{2}$ with $\bmod R=$ $P\left(A_{1}, A_{2}\right)$. Conversely, if a circular annulus $R$ separates $A_{1}$ from $A_{2}$, then

$$
P\left(A_{1}, A_{2}\right) \geq 2 \log \sinh \left(\frac{\bmod R}{2}\right) \geq \bmod R-2 \log (1+\sqrt{2}) .
$$

Proof. We first show the former part. By the Möbius invariance of the quantities involved, we may assume that $0 \in A_{1}$ and $\infty \in A_{2}$. For $a_{1} \in A_{1}, a_{2} \in A_{2}$ with $a_{1} \neq 0, a_{2} \neq \infty$, we have $\left|\operatorname{cr}\left(a_{1}, 0, \infty, a_{2}\right)\right|=\left|a_{2} / a_{1}\right|$. Since $\log ^{+}\left|\operatorname{cr}\left(a_{1}, 0, \infty, a_{2}\right)\right| \geq P\left(A_{1}, A_{2}\right)>0$, we obtain $\left|a_{2} / a_{1}\right|=\left|\operatorname{cr}\left(a_{1}, 0, \infty, a_{2}\right)\right| \geq e^{P\left(A_{1}, A_{2}\right)}>1$. Set $r_{1}=\max \left\{\left|a_{1}\right|: a_{1} \in A_{1}\right\}$ and $r_{2}=\min \left\{\left|a_{2}\right|: a_{2} \in A_{2}\right\}$. Then $r_{1}<r_{2}$ and the round annulus $R=\left\{z: r_{1}<|z|<r_{2}\right\}$ separates $A_{1}$ from $A_{2}$ and satisfies the inequality

$$
\bmod R=\log \frac{r_{2}}{r_{1}} \geq P\left(A_{1}, A_{2}\right) .
$$

Next we show the latter part. Suppose that a circular annulus $R$ separates $A_{1}$ from $A_{2}$. By a standard limiting process, we may assume, without loss of generality, that $\left(A_{1} \cup A_{2}\right) \cap \partial R=\emptyset$. Fix two pairs of distinct points $a_{1}, a_{1}^{\prime} \in A_{1}$ and $a_{2}, a_{2}^{\prime} \in A_{2}$. By using Möbius invariance of the quantities involved, we may further assume that $a_{2}^{\prime}=\infty$ and that $R$ is of the form $\mathbb{D} \backslash \overline{\mathbb{D}}(a, \rho)$ with $0 \leq a<1-\rho$. If we set $m=\bmod R$, then $R$ is Möbius equivalent to the round annulus $r:=e^{-m}<|z|<1$. Thus we can choose $0 \leq b<1$ so that $T_{b}(-r)=a-\rho$ and $T_{b}(r)=a+\rho$, where $T_{b}(z)=(z+b) /(1+b z)$. Since $a=\left(T_{b}(r)+T_{b}(-r)\right) / 2$ and $\rho=\left(T_{b}(r)-T_{b}(-r)\right) / 2$, we obtain

$$
\frac{1-a}{\rho}=\frac{1+b r^{2}}{(1+b) r}>\frac{1+r^{2}}{2 r} \text {. }
$$

Noting the inequalities $\left|a_{1}-a_{2}\right| \geq 1-(a+\rho)$ and $\left|a_{1}-a_{1}^{\prime}\right| \leq 2 \rho$, we obtain

$$
\left|\operatorname{cr}\left(a_{1}, a_{1}^{\prime}, a_{2}, \infty\right)\right|=\frac{\left|a_{1}-a_{2}\right|}{\left|a_{1}-a_{1}^{\prime}\right|} \geq \frac{1-a-\rho}{2 \rho}>\frac{1+r^{2}}{4 r}-\frac{1}{2}=\frac{(1-r)^{2}}{4 r}=\sinh ^{2} \frac{m}{2} .
$$

Hence, $P\left(A_{1}, A_{2}\right) \geq \log \sinh ^{2}(m / 2)=2 \log \sinh (m / 2)$. We note that $\sinh (m / 2) \geq 1$ if and only if $m \geq 2 \log (1+\sqrt{2})$. Since the function $f(x)=2 \log \sinh (x / 2)-x$ satisfies $f^{\prime}(x)=\operatorname{coth}(x / 2)-1>0$, we have $f(x) \geq f(2 \log (1+\sqrt{2}))=-2 \log (1+\sqrt{2})$ for $x \geq 2 \log (1+\sqrt{2})$. Thus the last inequality has been shown.

The following result roughly means that $1+Q(A)$ is comparable with $1 /$ sys $(X)$ for $X=\widehat{\mathbb{C}} \backslash A$.

Theorem 2.2. For an arbitrary hyperbolic punctured sphere $X=\widehat{\mathbb{C}} \backslash A$,

$$
Q(A) \leq \frac{\pi^{2}}{\operatorname{sys}(X)}
$$

Conversely, for every $\varepsilon \in\left(0, \pi^{2}\right)$, there exists a constant $\ell_{0}=\ell_{0}(\varepsilon) \in(0,1)$ such that the inequality

$$
\frac{\pi^{2}-\varepsilon}{\operatorname{sys}(X)}<Q(A)
$$


holds for any hyperbolic punctured sphere $X=\widehat{\mathbb{C}} \backslash A$ with sys $(X) \leq \ell_{0}$.

Proof. First we show the first inequality. We may assume that $Q(A)>0$. By definition, $Q(A)=P\left(A_{1}, A_{2}\right)$ for an admissible partition $A=A_{1} \sqcup A_{2}$. By the last lemma, there is a circular annulus $R$ separating $A_{1}$ from $A_{2}$ with $\bmod R=\log \frac{r_{2}}{r_{1}}=P\left(A_{1}, A_{2}\right)=Q(A)$. We may assume that $R$ is of the form $\left\{z: r_{1}<|z|<r_{2}\right\}$. Since the core curve $\gamma:|z|=\sqrt{r_{1} r_{2}}$ of $R$ has the hyperbolic length $\ell_{R}(\gamma)=\pi^{2} / \bmod R$ (see, for instance, [31, (4.2)]), we have

$$
Q(A)=\bmod R=\frac{\pi^{2}}{\ell_{R}(\gamma)} \leq \frac{\pi^{2}}{\ell_{X}(\gamma)} \leq \frac{\pi^{2}}{\operatorname{sys}(X)},
$$

as required.

We next show the second inequality. For a given $\varepsilon \in\left(0, \pi^{2}\right)$, choose an $0<\ell_{0} \leq 1$ so small that

$$
\arctan \left(1 / \sinh \ell_{0}\right)>\frac{\pi+\ell_{0}}{2}-\frac{\varepsilon}{2 \pi}
$$

Assume that sys $(X)<\ell_{0}$. Let $\gamma$ be a closed geodesic of length $\ell<\ell_{0}$ in $X$. It suffices to show that $\left(\pi^{2}-\varepsilon\right) / \ell \leq Q(A)$. Since $\ell_{0} \leq 1<\operatorname{arccosh} 3=2 \log (1+\sqrt{2})$, $\gamma$ is a power of a simple closed geodesic (see [39, Lemma 7 in p. 235]). We may assume that $\gamma$ is simple, namely, $\gamma$ is a Jordan curve in $X \subset \mathbb{C}$. Thus $\gamma$ divides $A$ into two parts, say, $A_{1}$ and $A_{2}$. Since $\gamma$ is homotopic to neither a point in $X$ nor a puncture, this partition is admissible. We now show that

$$
P\left(A_{1}, A_{2}\right)>\frac{\pi^{2}-\varepsilon}{\ell}
$$

which implies the required inequality because $Q(A) \geq P\left(A_{1}, A_{2}\right)$. Choose two pairs of points $a_{1}, a_{1}^{\prime} \in A_{1}$ and $a_{2}, a_{2}^{\prime} \in A_{2}$ so that $P\left(A_{1}, A_{2}\right)=\log ^{+}\left|\operatorname{cr}\left(a_{1}, a_{1}^{\prime}, a_{2}^{\prime}, a_{2}\right)\right|$. By Möbius invariance of the cross ratio, we may assume that $a_{1}^{\prime}=0$ and $a_{2}^{\prime}=\infty$. The collar lemma implies that there is an annulus $R$ in $X$ containing $\gamma$ as its core curve with $\bmod R>$ $2 \pi \arctan (1 / \sinh \ell) / \ell$ (Halpern [14, see also [31, Theorem 5.2]). By (2.6), we note that

$$
\bmod R>\frac{2 \pi \arctan (1 / \sinh \ell)}{\ell}>\frac{\pi^{2}-\varepsilon+\pi \ell_{0}}{\ell}>\frac{\pi^{2}-\varepsilon}{\ell}+\pi .
$$

A theorem of Avkhadiev-Wirths [5, Theorem 3.17] (see also [34, §3]) guarantees us the existence of a round annulus $R_{0}$ of the form $\left\{z \in \mathbb{C}: r_{1}<|z|<r_{2}\right\}$ contained in $R$ with $\bmod R_{0}=\log \left(r_{2} / r_{1}\right) \geq \bmod R-\pi$. Since $R_{0}$ separates $A_{1}$ from $A_{2}$, we have $\left|a_{1}\right| \leq r_{1}$ and $\left|a_{2}\right| \geq r_{2}$, and therefore, $\left|\operatorname{cr}\left(a_{1}, 0, \infty, a_{2}\right)\right|=\log \left|a_{2} / a_{1}\right| \geq \log \left(r_{2} / r_{1}\right)$. Hence,

$$
\log \left|\operatorname{cr}\left(a_{1}, a_{1}^{\prime}, a_{2}^{\prime}, a_{2}\right)\right| \geq \log \frac{r_{2}}{r_{1}} \geq \bmod R-\pi>\frac{\pi^{2}-\varepsilon}{\ell}
$$

and therefore $P\left(A_{1}, A_{2}\right)>\left(\pi^{2}-\varepsilon\right) / \ell$ as required.

We remark that the thrice-punctured sphere $\mathbb{C}_{0,1}=\widehat{\mathbb{C}} \backslash\{0,1, \infty\}$ is known to satisfy sys $\left(\mathbb{C}_{0,1}\right)=2 \log (1+\sqrt{2})=1.7627 \cdots>\ell_{0}$ (see [39]). From the above proof, we obtain also the following. 
Corollary 2.3. For a punctured spheres $X=\widehat{\mathbb{C}} \backslash A$ with $\operatorname{card}(A) \geq 3$, the inequalities

$$
\frac{C_{1}}{Q(A)+1} \leq \operatorname{sys}(X) \leq \frac{C_{2}}{Q(A)+1}
$$

hold, where $C_{1}$ and $C_{2}$ are absolute constants with $1<C_{1}<C_{2}<13.4$.

Proof. By the first inequality in Theorem 2.2 and the Schmutz inequality (2.4), we obtain

$$
\text { sys }(X)(Q(A)+1)<\pi^{2}+4 \log (1+\sqrt{2}) \approx 13.3951 .
$$

Thus the right-hand side inequality has been shown. To show the other one, we take $\ell_{0}=1$ so that

$$
\varepsilon=2 \pi\left[\left(\pi+\ell_{0}\right) / 2-\arctan \left(1 / \sinh \ell_{0}\right)=\pi(\pi+1)-2 \pi \arctan \left(1 /\left(e-e^{-1}\right)\right) \approx 8.58 .\right.
$$

If sys $(X) \leq 1$, the last theorem yields

$$
\text { sys }(X)(Q(A)+1)>\operatorname{sys}(X) Q(A)>\pi^{2}-\epsilon>1.28 \text {. }
$$

Otherwise, sys $(X)(Q(A)+1) \geq \operatorname{sys}(X)>1$. Therefore, we have sys $(X)(Q(A)+1))>1$ at any event.

In the inequalities of the last corollary, we cannot replace the denominator $Q(A)+1$ by $Q(A)$ in general. Indeed, $Q(A)$ may be very small even when sys $(X)$ is not so small as the following example shows.

Example 2.4. Let $X=\widehat{\mathbb{C}} \backslash A$, where $A=\{0,1, a, \infty\}$ for $a \in \mathbb{C} \backslash\{0,1, \infty\}$. In view of the formula $\operatorname{cr}(0,1, a, \infty)=a$, we have

$$
Q(A)=\widehat{Q}(A)=\max \{|\log | a||,|\log | 1-a||,|\log | 1-1 / a||\} .
$$

We observe that $Q(A)=\widehat{Q}(A) \rightarrow+\infty$ precisely when $a$ approaches the boundary of $\mathbb{C}_{0,1}$. For instance, $Q(A)=\log |a|$ when $a$ lies in the set $\{z \in \widehat{\mathbb{C}}: \operatorname{Re} z \geq 1 / 2,|z-1| \geq 1\}$. In particular, $Q(A)=0$ when $a=e^{ \pm \pi i / 3}$.

We next consider a finite subset $A$ of a circle $C$ in $\widehat{\mathbb{C}}$. Suppose that $P\left(A_{1}, A_{2}\right)>0$ for an admissible partition $A=A_{1} \sqcup A_{2}$. By the former part of Lemma 2.1, we observe that $A_{1}$ does not separate $A_{2}$ in $C$. Therefore, we may assume that $C=\mathbb{R} \cup\{\infty\}$ and $a_{1}<a_{2}$ whenever $a_{1} \in A_{1}$ and $a_{2} \in A_{2}$. By (2.3), we have

$$
\operatorname{cr}\left(a_{1}, a_{1}^{\prime}, a_{2}, a_{2}^{\prime}\right)=1-\operatorname{cr}\left(a_{1}^{\prime}, a_{1}, a_{2}, a_{2}^{\prime}\right)=1+\frac{\left(a_{2}-a_{1}^{\prime}\right)\left(a_{2}^{\prime}-a_{1}\right)}{\left(a_{1}^{\prime}-a_{1}\right)\left(a_{2}^{\prime}-a_{2}\right)}>1
$$

for $a_{1}, a_{1}^{\prime} \in A_{1}$ and $a_{2}, a_{2}^{\prime} \in A_{2}$ with $a_{1}<a_{1}^{\prime}$ and $a_{2}<a_{2}^{\prime}$. Moreover, $\operatorname{cr}\left(a_{1}, a_{1}^{\prime}, a_{2}, a_{2}^{\prime}\right)$ is minimised when $a_{2}=\min A_{2}$ and $a_{2}^{\prime}=\max A_{2}$ for a fixed pair $\left(a_{1}, a_{1}^{\prime}\right)$. Indeed, this can easily be checked by normalising $a_{1}=0$ and $a_{1}^{\prime}=1$. Similarly, we observe that $\operatorname{cr}\left(a_{1}, a_{1}^{\prime}, a_{2}, a_{2}^{\prime}\right)$ is minimised when $a_{1}=\min A_{1}$ and $a_{1}^{\prime}=\max A_{1}$ for a fixed pair $\left(a_{2}, a_{2}^{\prime}\right)$. In view of (2.2), we summarise these observations as follows.

Proposition 2.5. Let $A_{1}$ and $A_{2}$ be finite subsets of $\mathbb{R}$ such that $\operatorname{card}\left(A_{j}\right) \geq 2, j=1,2$, and $a_{1}<a_{2}$ for $a_{1} \in A_{1}$ and $a_{2} \in A_{2}$. Then

$$
P\left(A_{1}, A_{2}\right)=\log ^{+}\left\{\operatorname{cr}\left(\min A_{1}, \max A_{1}, \min A_{2}, \max A_{2}\right)-1\right\} .
$$


The following example shows that $Q(A)$ and $\widehat{Q}(A)$ are not comparable.

Example 2.6. Let $A=\{0,1, \ldots, n\}$ for $n \geq 3$. If $A_{1}=\{k+1, k+2, \ldots, l-1, l\}$ for some $1 \leq k+1<l<n$, then $\operatorname{cr}(k, l+1, k+1, l)=-1 /\left\{(l-k)^{2}-1\right\}$ and thus $P\left(A_{1}, A_{2}\right)=0$. If $A_{1}=\{0,1, \ldots, k\}$ for some $1 \leq k \leq n-2$, then by the above proposition, $P\left(A_{1}, A_{2}\right)=\log ^{+}(\operatorname{cr}(0, k, k+1, n)-1)=\log ^{+} \frac{n}{k(n-k-1)}$, which is maximised when $k=1$ or $k=n-2$. Thus we have $Q(A)=\log \frac{n}{n-2}$. On the other hand, since $\left|\left(a_{3}-a_{1}\right)\left(a_{4}-a_{2}\right)\right| \geq$ $\max \left\{n(n-2),(n-1)^{2}\right\}=(n-1)^{2}$ and $\left|\left(a_{2}-a_{1}\right)\left(a_{4}-a_{3}\right)\right| \geq 1$ for any distinct $a_{1}, a_{2}, a_{3}, a_{4}$ in $A$, we obtain $\left|\operatorname{cr}\left(a_{1}, a_{2}, a_{3}, a_{4}\right)\right| \leq(n-1)^{2}$, where equality holds for $\left(a_{1}, a_{2}, a_{3}, a_{4}\right)=$ $(0,1, n-1, n)$. Therefore, $\widehat{Q}(A)=2 \log (n-1)$. This example shows that $Q(A) \rightarrow 0$ whereas $\widehat{Q}(A) \rightarrow+\infty$ as $n \rightarrow \infty$. By Theorem [2.2, $1+Q(A)$ is comparable with $1 /$ sys $(X)$ but we cannot replace $Q(A)$ by $\widehat{Q}(A)$ in the theorem.

\section{Construction of a distance}

Suppose that $f:[0, \pi] \rightarrow(0, \infty)$ is a continuous increasing subadditive function with $f(0)=0$. Here, subadditivity means that $f\left(t_{1}+t_{2}\right) \leq f\left(t_{1}\right)+f\left(t_{2}\right)$ whenever $t_{1}, t_{2}, t_{1}+t_{2} \in$ $[0, \pi]$. We then define a function $D=D^{f}$ on $\overline{\mathbb{D}}^{*}(0,1 / e)^{2}$ by

$$
\begin{aligned}
D^{f}\left(z_{1}, z_{2}\right) & =\frac{f(\theta)}{\max \left\{\log \left(1 /\left|z_{1}\right|\right), \log \left(1 /\left|z_{2}\right|\right)\right\}}+\left|\log \frac{\log \left(1 /\left|z_{1}\right|\right)}{\log \left(1 /\left|z_{2}\right|\right)}\right| \\
& =\frac{f(\theta)}{\max \left\{\tau_{1}, \tau_{2}\right\}}+\left|\log \tau_{1}-\log \tau_{2}\right|
\end{aligned}
$$

for $z_{1}, z_{2} \in \overline{\mathbb{D}}^{*}(0,1 / e)$, where $\theta=\left|\arg \left(z_{2} / z_{1}\right)\right|=2 \arcsin \left(\left|\zeta_{1}-\zeta_{2}\right| / 2\right) \in[0, \pi], \zeta_{l}=$ $z_{l} /\left|z_{l}\right|, \tau_{l}=-\log \left|z_{l}\right|(l=1,2)$. Note that $D^{f}\left(z_{1}, z_{2}\right)=f(\theta)$ for $\left|z_{1}\right|=\left|z_{2}\right|=e^{-1}$. Our construction will be based on the following lemma.

Lemma 3.1. Let $L_{1}$ and $L_{2}$ be positive numbers and let $f:[0, \pi] \rightarrow(0, \infty)$ be a continuous increasing subadditive function satisfying $L_{1} t \leq f(t) \leq L_{2} t$ for $0 \leq t \leq \pi$. Then

$$
2 L_{1} h_{\mathbb{D}^{*}}\left(z_{1}, z_{2}\right) \leq D^{f}\left(z_{1}, z_{2}\right) \leq M_{0} L_{2} h_{\mathbb{C}_{0,1}}\left(z_{1}, z_{2}\right), \quad 0<\left|z_{1}\right|,\left|z_{2}\right| \leq e^{-1},
$$

where $M_{0}$ is a constant with $M_{0}<24$. If $f \leq 2$ in addition, then $D^{f}\left(z_{1}, z_{2}\right)$ satisfies the triangle inequality on $0<|z| \leq e^{-1}$.

The first part is proved in [36] for $f(t)=2 \sin (t / 2)$ and $f(t)=\mathrm{id}(t)=t$. The second part is also proved for $f(t)=2 \sin (t / 2)$ in [36]. We include an outline of the proof here for the convenience of the reader. For details, see [36].

For instance, if $f$ is a continuously differentiable, increasing, and concave function in $[0, \pi]$ with $f(0)=0$, then $f(t) / t$ is non-increasing and $f$ is subadditive. In particular, the inequalities

$$
\frac{f(\pi)}{\pi} \leq \frac{f(t)}{t} \leq f^{\prime}(0), \quad 0<t \leq \pi
$$

hold. Thus we can take $L_{1}=f(\pi) / \pi$ and $L_{2}=f^{\prime}(0)$ in this case.

Proof of Lemma 3.1. For the first part, we note that the inequalities $L_{1} D^{\text {id }}\left(z_{1}, z_{2}\right) \leq$ $D^{f}\left(z_{1}, z_{2}\right) \leq L_{2} D^{\text {id }}\left(z_{1}, z_{2}\right)$ hold. Since the inequalities $2 h_{\mathbb{D}^{*}}\left(z_{1}, z_{2}\right) \leq D^{\text {id }}\left(z_{1}, z_{2}\right) \leq$ $M_{0} h_{\mathbb{C}_{0,1}}\left(z_{1}, z_{2}\right)$ are already shown in [36], the required inequalities follow. 
We next show the triangle inequality $\Delta:=D\left(z_{1}, z\right)+D\left(z, z_{2}\right)-D\left(z_{1}, z_{2}\right) \geq 0$ under the assumption $f \leq 2$. The most delicate case is when $1 \leq \tau_{2} \leq \tau_{1} \leq \tau$, where $\tau_{l}=$ $-\log \left|z_{l}\right|(l=1,2)$ and $\tau=-\log |z|$. The other cases can be handled similarly or even more easily, and thus, will be omitted. Let $\theta=\left|\arg \left(z_{2} / z_{1}\right)\right|, \theta_{l}=\left|\arg \left(z_{l} / z\right)\right| \in[0, \pi](l=1,2)$. Since $\theta \leq \theta_{1}+\theta_{2}$, it is easy to check that the inequality $f(\theta) \leq f\left(\theta_{1}\right)+f\left(\theta_{2}\right)$ holds. Thus,

$$
\begin{aligned}
\Delta & =\frac{f\left(\theta_{1}\right)+f\left(\theta_{2}\right)}{\tau}+\log \frac{\tau}{\tau_{1}}+\log \frac{\tau}{\tau_{2}}-\frac{f(\theta)}{\tau_{1}}-\log \frac{\tau_{1}}{\tau_{2}} \\
& \geq \frac{f(\theta)}{\tau}-\frac{f(\theta)}{\tau_{1}}+2 \log \tau-2 \log \tau_{1}=: g(\tau) .
\end{aligned}
$$

Since $g^{\prime}(\tau)=[2 \tau-f(\theta)] / \tau^{2} \geq[2-f(\theta)] / \tau^{2} \geq 0$, one has $\Delta \geq g(\tau) \geq g\left(\tau_{1}\right)=0$.

Remark. We remark that if "maximum" is replaced by "minimum" in the definition of the function $D^{f}\left(z_{1}, z_{2}\right)$, then the corresponding quantity does not necessarily satisfy the triangle inequality.

We enumerate the elements of $A$ as $a_{1}, \ldots, a_{n}$ so that $a_{n}=\infty$. Set

$$
\tilde{\rho}_{j}= \begin{cases}\min _{1 \leq k<n, k \neq j}\left|a_{k}-a_{j}\right| & \text { for } j=1,2, \ldots, n-1, \\ \max _{1 \leq k<n}\left|a_{k}\right| & \text { for } j=n,\end{cases}
$$

$\rho_{j}=\tilde{\rho}_{j} / e$ for $1 \leq j<n$ and $\rho_{n}=e \tilde{\rho}_{n}$. We next consider the sets $E_{j}=\overline{\mathbb{D}}\left(a_{j}, \rho_{j}\right)$ for $1 \leq j<n$ and $E_{n}=\left\{z \in \widehat{\mathbb{C}}:|z| \geq \rho_{n}\right\}$. Furthermore, set $E_{j}^{*}=E_{j} \backslash\left\{a_{j}\right\}$ and $W=\widehat{\mathbb{C}} \backslash\left(E_{1} \cup \cdots \cup E_{n}\right)$. Note that $E_{1}, \ldots, E_{n}$ are disjoint. Since $0 \in A$, we observe that $e \rho_{j}=\tilde{\rho}_{j} \leq\left|a_{j}\right| \leq \tilde{\rho}_{n}=\rho_{n} / e$ for $1 \leq j<n$. In particular,

$$
\max _{1 \leq j<n} \rho_{j} \leq e^{-2} \rho_{n}
$$

We set

$$
\rho_{\min }=\min _{1 \leq j \leq n} \rho_{j} \quad \text { and } \quad \rho_{\max }=\max _{1 \leq j \leq n} \rho_{j}=\rho_{n} .
$$

Suppose that $\mu\left(z_{1}, z_{2}\right)$ is a distance function on $X$ such that

$$
\mu\left(z_{1}, z_{2}\right)=S_{j} f_{j}(\theta) \quad \text { for } z_{1}, z_{2} \in \partial E_{j}, j=1, \ldots, n,
$$

where $\theta=\left|\arg \left(\left(z_{2}-a_{j}\right) /\left(z_{1}-a_{j}\right)\right)\right| \in[0, \pi]$ for $j<n$ and $\theta=\left|\arg \left(z_{2} / z_{1}\right)\right| \in[0, \pi]$ for $j=n, f_{j}:[0, \pi] \rightarrow[0,2]$ is a continuous increasing subadditive function with $f_{j}(0)=0$ and $S_{j}$ is a positive constant for each $j$. We note that by (3.2) the function $\mu\left(z_{1}, z_{2}\right)$ is rotationally invariant on $\partial E_{j}$ about $a_{j}$.

Example 3.2. When $\mu\left(z_{1}, z_{2}\right)$ is the euclidean distance $\left|z_{1}-z_{2}\right|$, we can choose $f_{j}(t)=$ $2 \sin (t / 2)$ and $S_{j}=\rho_{j}$ in the above construction. 
Then, we define a function $D_{j}\left(z_{1}, z_{2}\right)$ on $E_{j}^{*} \times E_{j}^{*}$ by

$$
D_{j}\left(z_{1}, z_{2}\right)= \begin{cases}S_{j} D^{f_{j}}\left(\frac{z_{1}-a_{j}}{\tilde{\rho}_{j}}, \frac{z_{2}-a_{j}}{\tilde{\rho}_{j}}\right) & \text { if } 1 \leq j<n, \\ S_{n} D^{f_{n}}\left(\frac{\tilde{\rho}_{n}}{z_{1}}, \frac{\tilde{\rho}_{n}}{z_{2}}\right) & \text { if } j=n,\end{cases}
$$

for $z_{1}, z_{2} \in E_{j}^{*}$. Then $D_{j}\left(z_{1}, z_{2}\right)=\mu\left(z_{1}, z_{2}\right)$ for $z_{1}, z_{2} \in \partial E_{j}$. We now apply the following lemma with the choice $Y_{0}=W, d_{0}=\mu, Y_{j}=E_{j}^{*}, d_{j}=D_{j}(1 \leq j \leq n)$ to construct a new distance $d$ on $X$. We will denote by $d_{X}$ this distance $d$ constructed in this way with $\mu, f_{j}, S_{j}(j=1, \ldots, n)$. The same construction was given in [36] when $\mu\left(z_{1}, z_{2}\right)=\left|z_{1}-z_{2}\right|$.

Lemma 3.3. Let $X$ be a set and assume that $X=Y_{0} \cup Y_{1} \cup \cdots \cup Y_{n}$. Let $d_{j}$ be a distance function on $Y_{j}$ for $j=0,1, \ldots, n$. Suppose that $Y_{j} \cap Y_{k}=\emptyset$ for $j \neq k$ with $1 \leq j, k \leq n$, that $d_{0}\left(x_{1}, x_{2}\right)=d_{j}\left(x_{1}, x_{2}\right)$ for $x_{1}, x_{2} \in Z_{j}=Y_{0} \cap Y_{j}, j=1, \ldots, n$, and that each $Z_{j}$ is non-empty and closed in the both metric spaces $\left(Y_{0}, d_{0}\right)$ and $\left(Y_{j}, d_{j}\right)$. We define $d\left(x_{1}, x_{2}\right)$ for $x_{1}, x_{2} \in X$ to be

$$
\begin{cases}d_{j}\left(x_{1}, x_{2}\right) & \text { for } x_{1}, x_{2} \in Y_{j}^{\prime} \text { with } 0 \leq j \leq n, \\ \inf _{\zeta \in Z_{j}}\left\{d_{j}\left(x_{1}, \zeta\right)+d_{0}\left(\zeta, x_{2}\right)\right\} & \text { for } x_{1} \in Y_{j}, x_{2} \in Y_{0} \text { with } j \neq 0, \\ \inf _{\zeta \in Z_{j}}\left\{d_{0}\left(x_{1}, \zeta\right)+d_{j}\left(\zeta, x_{2}\right)\right\} & \text { for } x_{1} \in Y_{0}, x_{2} \in Y_{j} \text { with } j \neq 0, \\ \inf _{\substack{\zeta_{1} \in Z_{j} \\ \zeta_{2} \in Z_{k}}}\left\{d_{j}\left(x_{1}, \zeta_{1}\right)+d_{0}\left(\zeta_{1}, \zeta_{2}\right)+d_{k}\left(\zeta_{2}, x_{2}\right)\right\} & \text { for } x_{1} \in Y_{j}, x_{2} \in Y_{k} \text { with } 0 \neq j \neq k \neq 0,\end{cases}
$$

where $Y_{j}^{\prime}=Y_{j} \backslash Z_{j}$ for $j=1, \ldots, n$ and $Y_{0}^{\prime}=Y_{0} \backslash\left(Z_{1} \cup \cdots \cup Z_{n}\right)$. Then $d$ is a distance function on $X$ such that $d\left(x_{1}, x_{2}\right)=d_{j}\left(x_{1}, x_{2}\right)$ for $x_{1}, x_{2} \in Y_{j}, j=0,1, \ldots, n$.

Proof. First we check that $d=d_{j}$ on $Y_{j} \times Y_{j}$. For instance, we assume that $x_{1} \in Y_{j}^{\prime}$ and $x_{2} \in Z_{j}$ for some $j=1, \ldots, n$. Letting $\zeta=x_{2}$ in the second case of the definition of $d\left(x_{1}, x_{2}\right)$, we see that $d\left(x_{1}, x_{2}\right) \leq d_{j}\left(x_{1}, x_{2}\right)$. On the other hand, for any $\zeta \in Z_{j}$, by the triangle inequality and the assumption, we have

$$
d_{j}\left(x_{1}, \zeta\right)+d_{0}\left(\zeta, x_{2}\right)=d_{j}\left(x_{1}, \zeta\right)+d_{j}\left(\zeta, x_{2}\right) \geq d_{j}\left(x_{1}, x_{2}\right),
$$

which implies $d\left(x_{1}, x_{2}\right) \geq d_{j}\left(x_{1}, x_{2}\right)$. Thus we have $d\left(x_{1}, x_{2}\right)=d_{j}\left(x_{1}, x_{2}\right)$. The other cases can be handled similarly. We thus checked the last assertion.

We next show that $d$ is a distance function on $X$. The symmetricity $d\left(x_{1}, x_{2}\right)=d\left(x_{2}, x_{1}\right)$ and nonnegativity are obvious by definition. We now show that $d\left(x_{1}, x_{2}\right)=0$ only if $x_{1}=x_{2}$. Suppose that $d\left(x_{1}, x_{2}\right)=0$ for a pair of points $x_{1}, x_{2}$ in $X$. When $x_{1}$ and $x_{2}$ are contained in a common $Y_{j}$, then $d\left(x_{1}, x_{2}\right)=d_{j}\left(x_{1}, x_{2}\right)=0$ implies $x_{1}=x_{2}$ certainly. We now consider, for example, the case when $x_{1} \in Y_{j}^{\prime}$ and $x_{2} \in Y_{0}$ for some $j \neq 0$. Then, by definition,

$$
0=d\left(x_{1}, x_{2}\right) \geq \inf _{\zeta \in Z_{j}} d_{j}\left(x_{1}, \zeta\right)
$$

This implies that $x_{1}$ is contained in the closure of $Z_{j}$, which is a contradiction because $Z_{j}$ is closed in $Y_{j}$. The other cases can be excluded in a similar way. Hence, we conclude that $d\left(x_{1}, x_{2}\right)=0$ implies $x_{1}=x_{2}$. 
We finally check the triangle inequality. As a sample case, we assume that $x_{1} \in Y_{j}, x_{2} \in$ $Y_{0}, x_{3} \in Y_{k}$ with $j \neq 0 \neq k \neq j$. Then

$$
\begin{aligned}
d\left(x_{1}, x_{2}\right)+d\left(x_{2}, x_{3}\right) & =\inf _{\zeta_{1} \in Z_{j}, \zeta_{2} \in Z_{k}}\left\{d_{j}\left(x_{1}, \zeta_{1}\right)+d_{0}\left(\zeta_{1}, x_{2}\right)+d_{0}\left(x_{2}, \zeta_{2}\right)+d_{k}\left(\zeta_{2}, x_{3}\right)\right\} \\
& \geq \inf _{\zeta_{1} \in Z_{j}, \zeta_{2} \in Z_{k}}\left\{d_{j}\left(x_{1}, \zeta_{1}\right)+d_{0}\left(\zeta_{1}, \zeta_{2}\right)+d_{k}\left(\zeta_{2}, x_{3}\right)\right\} \\
& =d\left(x_{1}, x_{3}\right) .
\end{aligned}
$$

We can deal with the other cases similarly.

We now state a general result. This was already shown in [36] under the situation of Example 3.2. Indeed, when $\mu\left(z_{1}, z_{2}\right)=\left|z_{1}-z_{2}\right|$, a similar quantity $e_{X}\left(z_{1}, z_{2}\right)$ is defined without taking the infima and a comparison result is given for it as Theorem 5.1 in [36]. Since its proof is tedious, we restrict our attention to $d_{X}\left(z_{1}, z_{2}\right)$ for simplicity in the present note.

Theorem 3.4. Let $X=\widehat{\mathbb{C}} \backslash A$ be an $n$-times punctured sphere with $n \geq 3$ and $X \subset$ $\mathbb{C} \backslash\{0,1\}$. Let $d_{X}$ be the distance on $X$ constructed in Lemma 3.3 with a distance $\mu\left(z_{1}, z_{2}\right)$ satisfying the condition (3.2) with $f_{j}:[0, \pi] \rightarrow[0,2], S_{j}>0,(j=1, \ldots, n)$. Suppose that $L_{1} t \leq f_{j}(t) \leq L_{2} t$ for $0 \leq t \leq \pi$ and $j=1, \ldots, n$ and that $K_{1} h_{X}\left(z_{1}, z_{2}\right) \leq \mu\left(z_{1}, z_{2}\right) \leq$ $K_{2} h_{X}\left(z_{1}, z_{2}\right)$ for $z_{1}, z_{2} \in W=X \backslash\left(E_{1}^{*} \cup \cdots \cup E_{n}^{*}\right)$. Then the distance $d_{X}\left(z_{1}, z_{2}\right)$ on $X$ satisfies the inequalities

$$
B_{1} h_{X}\left(z_{1}, z_{2}\right) \leq d_{X}\left(z_{1}, z_{2}\right) \leq B_{2} h_{X}\left(z_{1}, z_{2}\right), \quad z_{1}, z_{2} \in X
$$

where

$$
B_{1}=\min \left\{2 S_{\min } L_{1}, K_{1}\right\} \quad \text { and } \quad B_{2}=\max \left\{S_{\max } M_{0} L_{2}, K_{2}\right\}
$$

and $S_{\max }=\max \left\{S_{1}, \ldots, S_{n}\right\}, S_{\min }=\min \left\{S_{1}, \ldots, S_{n}\right\}, M_{0}<24$.

Proof. Since the method is almost the same as in [36, Theorem 5.1], we give only a sketchy proof. As a typical case, we consider the situation that $z_{1} \in E_{j}^{*}$ and $z_{2} \in W$. Let $\alpha$ be a shortest hyperbolic geodesic joining $z_{1}$ and $z_{2}$ in $X$. Then $h_{X}\left(z_{1}, z_{2}\right)=h_{X}\left(z_{1}, \zeta_{0}\right)+$ $h_{X}\left(\zeta_{0}, z_{2}\right)$ for the first hitting point $\zeta_{0}$ of $\alpha$ to $\partial E_{j}$ starting from $z_{1}$. We first assume that $j \neq n$. Choose $a_{k}$ so that $\tilde{\rho}_{j}=\left|a_{k}-a_{j}\right|$ and set $X_{1}=\mathbb{C} \backslash\left\{a_{j}, a_{k}\right\}$. By Lemma 3.1, we obtain

$$
D_{j}\left(z_{1}, \zeta_{0}\right) \leq S_{j} M_{0} L_{2} h_{X_{1}}\left(z_{1}, \zeta_{0}\right) \leq S_{j} M_{0} L_{2} h_{X}\left(z_{1}, \zeta_{0}\right)
$$

Next we assume that $j=n$. Choose $a_{k}$ so that $\tilde{\rho}_{n}=\left|a_{k}\right|$ and set $g(z)=a_{k} / z$. Then, by Lemma 3.1 again, we have

$$
D_{n}\left(z_{1}, \zeta_{0}\right) \leq S_{n} M_{0} L_{2} h_{\mathbb{C}_{0,1}}\left(g\left(z_{1}\right), g\left(\zeta_{0}\right)\right) \leq S_{n} M_{0} L_{2} h_{X}\left(z_{1}, \zeta_{0}\right) .
$$

Since $\mu\left(\zeta_{0}, z_{2}\right) \leq K_{2} h_{X}\left(\zeta_{0}, z_{2}\right)$ by assumption, we obtain

$$
d_{X}\left(z_{1}, z_{2}\right) \leq D_{j}\left(z_{1}, \zeta_{0}\right)+\mu\left(\zeta_{0}, z_{2}\right) \leq B_{2} h_{X}\left(z_{1}, z_{2}\right) .
$$

On the other hand, by definition, $d_{X}\left(z_{1}, z_{2}\right)=D_{j}\left(z_{1}, \zeta\right)+\mu\left(\zeta, z_{2}\right)$ for some $\zeta \in \partial E_{j}$. By Lemma 3.1, in a similar way as above, we obtain $D_{j}\left(z_{1}, \zeta\right) \geq 2 S_{j} L_{1} h_{X}\left(z_{1}, \zeta\right)$. Thus,

$$
d_{X}\left(z_{1}, z_{2}\right) \geq 2 S_{\min } L_{1} h_{X}\left(z_{1}, \zeta\right)+K_{1} h_{X}\left(\zeta, z_{2}\right) \geq B_{1} h_{X}\left(z_{1}, z_{2}\right) .
$$

Thus the proof is complete. 
As an example, we apply the last theorem to Example 3.2. We note here that one can choose $L_{1}=2 / \pi, L_{2}=1, K_{1}=2 \rho_{\min } / \pi$ and

$$
K_{2}=\max \left\{2 \rho_{n} e^{-K_{0}}\left(C_{0}-K_{0}-1+\log \left[\rho_{n} / \rho_{\min }\right]\right), 6 \rho_{n}\left(C_{0}+\log \left[3 \rho_{n}\right]\right)\right\},
$$

where $C_{0}=1 /\left(2 \lambda_{\mathbb{C}_{0,1}}(-1)\right)=\Gamma(1 / 4)^{4} / 4 \pi^{2} \approx 4.37688$ and $K_{0} \approx 0.846666$ (see the proof of Theorem 5.1 in [36] for details). Thus, in view of (3.1), we obtain the following result, which is essentially contained in [36].

Corollary 3.5. Let $X=\widehat{\mathbb{C}} \backslash\left\{a_{1}, \ldots, a_{n}\right\}$ be an $n$-times punctured sphere with $a_{1}=$ $0, a_{n}=\infty$ and let $d_{X}$ be the distance on $X$ constructed above for the choices $\mu\left(z_{1}, z_{2}\right)=$ $\left|z_{1}-z_{2}\right|, f_{j}(t)=2 \sin (t / 2)$ and $S_{j}=\rho_{j}$ for $j=1, \ldots, n$. Then

$$
B_{1} h_{X}\left(z_{1}, z_{2}\right) \leq d_{X}\left(z_{1}, z_{2}\right) \leq B_{2} h_{X}\left(z_{1}, z_{2}\right), \quad z_{1}, z_{2} \in X
$$

where

$$
B_{1}=\frac{2 \rho_{\min }}{\pi} \quad \text { and } \quad B_{2}=\max \left\{M_{0} \rho_{n}, K_{2}\right\}
$$

$K_{2}$ is given in (3.3) and $\rho_{\min }=\min \left\{\rho_{1}, \ldots, \rho_{n}\right\}$.

\section{QUASIHYPERBOLIC TYPE CONSTRUCTIONS}

Let $X=\widehat{\mathbb{C}} \backslash A$ be an $n$-times punctured sphere as before, where $A$ is a finite subset of $\widehat{\mathbb{C}}$ with $0,1, \infty \in A$. We recall that $\delta_{X}(z)$ denotes the euclidean distance from $z \in X$ to the boundary $\partial X=A$. For all $z$ in an open dense subset of $X$, there is only one $a \in A$ such that $\delta_{X}(z)=|z-a|$. For $a \in A$, we set $V_{a}=\{z \in \mathbb{C}:|z-a| \leq|z-b|$ for any $b \in A\}$. Note that each $V_{a}$ is a closed convex set and that $V_{\infty}=\emptyset$. Then we obtain a partition of $\mathbb{C}$ into $V_{a}, a \in A$, which is known as the Voronoi diagram. Each $V_{a}$ is called a Voronoi cell and the point $a$ is called the nucleus of $V_{a}$. Since $\mathbb{C}$ is tessellated by $V_{a}$ 's, some of them are unbounded. It is known that $V_{a}$ is unbounded if and only if $a$ lies on the boundary of the convex hull of $A \backslash\{\infty\}$. See Figure 1 (a). We refer to [38] for the relation between the quasihyperbolic metric and the Voronoi diagram. Set $\delta_{a}(z)=|z-a|$. Then $\delta_{X}(z)=\min _{a \in A} \delta_{a}(z)$. Since $\delta_{X}(z)=\delta_{a}(z)$ for $z \in V_{a}$, we see that the function $\delta_{X}\left(r e^{i \theta}\right)$ is not of class $C^{1}$ in $\theta \in \mathbb{R}$ (and thus, not rotationally invariant) for large enough $r>0$. Therefore, the $j$-distance (see [4] for instance)

$$
j_{X}\left(z_{1}, z_{2}\right)=\log \left(1+\frac{\left|z_{1}-z_{2}\right|}{\min \left\{\delta_{X}\left(z_{1}\right), \delta_{X}\left(z_{2}\right)\right\}}\right)
$$

does not satisfy the condition (3.2).

Therefore, we are led to a slight modification of the function $\delta_{X}$ in the following way:

$$
\hat{\delta}_{X}(z)=\min \left\{\delta_{X}(z),|z| / 2\right\} .
$$

Here we recall that the set $A$ of the punctures contains 0 and $\infty$. Note that $\delta_{X}(z) / 2 \leq$ $\hat{\delta}_{X}(z) \leq \delta_{X}(z)$ for $z \in X$. In other words, $\hat{\delta}_{X}(z)=\min _{a \in A} \hat{\delta}_{a}(z)$, where

$$
\hat{\delta}_{a}(z)= \begin{cases}|z-a| & \text { if } a \neq 0, \\ |z| / 2 & \text { if } a=0, \\ +\infty & \text { if } a=\infty .\end{cases}
$$


Then the modified Voronoi diagram (with respect to the origin) is defined by setting

$$
\hat{V}_{a}=\left\{z \in \mathbb{C}: \hat{\delta}_{a}(z) \leq \hat{\delta}_{b}(z) \text { for any } b \in A\right\}
$$

as the modified Voronoi cell for $a \in A$. For $a, b \neq 0$, the set $H_{a, b}=\left\{z: \hat{\delta}_{a}(z) \leq \hat{\delta}_{b}(z)\right\}$ is the half-plane containing $a$ and bounded by the bisector of the segment joining the two points $a$ and $b$. It is easy to see that $H_{a, 0}$ is the Apollonian disk $\{z:|z-a| \leq|z| / 2\}$; namely, $\{z:|z-4 a / 3| \leq 2|a| / 3\}$. Therefore, for $a \in A \backslash\{0, \infty\}, \hat{V}_{a}$ is the intersection of a (possibly unbounded) convex polygon with the disk $H_{a, 0}$ and, in particular, a compact convex set. On the other hand, $\hat{V}_{0}$ is a (possibly disconnected, non-convex) unbounded closed set. By definition, we have $\hat{\delta}_{X}(z)=\hat{\delta}_{a}(z)$ for $z \in \hat{V}_{a}, a \in A$. See Figure 1 (b) for the modified Voronoi diagram.

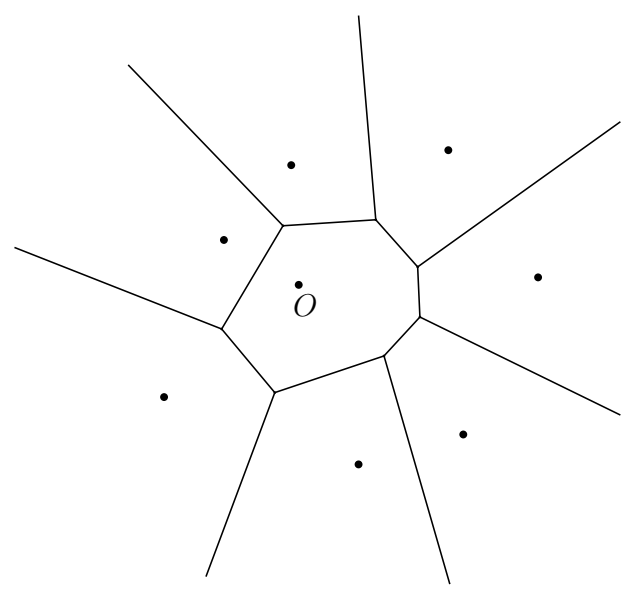

(a)

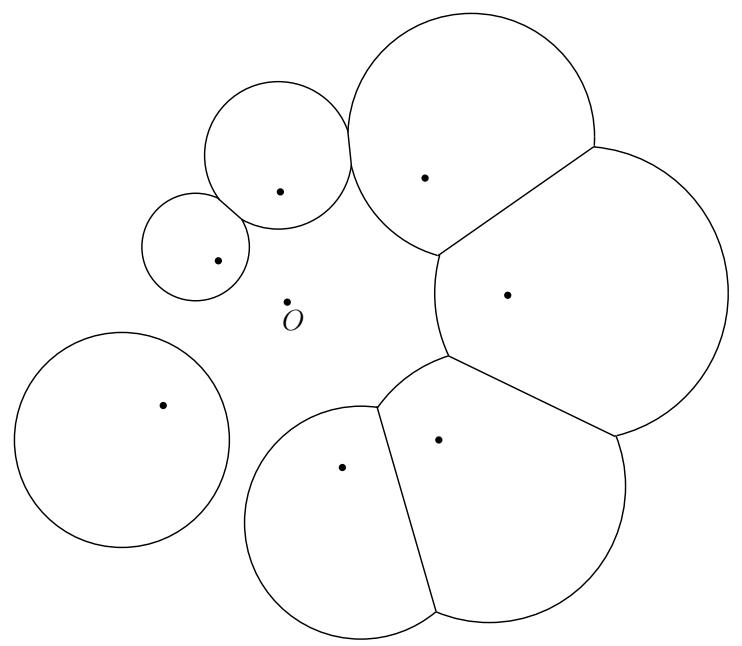

(b)

FiguRE 1. Voronoi diagram (a) and modified Voronoi diagram (b)

We enumerate $A$ as $a_{1}, \ldots, a_{n}$ so that $a_{1}=0$ and $a_{n}=\infty$, as before. Then we have the next result.

Lemma 4.1. $\hat{\delta}_{X}(z)=|z| / 2$ if either $z \in E_{1}^{*}$ or $z \in E_{n}^{*}$. For $z \in E_{j}^{*}$ with $1<j<n$, $\hat{\delta}_{X}(z)=\left|z-a_{j}\right|$.

Proof. Let $z \in E_{n}$ and $a$ be a finite point in $A$. Since $|a| \leq 1 / \tilde{\rho}_{n}=1 / e \rho_{n}$, we obtain $|z-a| \geq|z|-1 / e \rho_{n} \geq|z| / 2$. Thus $\delta_{X}(z) \geq|z| / 2$ for $z \in E_{n}$. The other cases can also be checked similarly.

Applying this idea, we define a modified version of the $j$-distance as follows

$$
\hat{j}_{X}\left(z_{1}, z_{2}\right)=\log \left(1+\frac{\left|z_{1}-z_{2}\right|}{\min \left\{\hat{\delta}_{X}\left(z_{1}\right), \hat{\delta}_{X}\left(z_{2}\right)\right\}}\right) .
$$

Noting the elementary inequality $\left|\delta_{X}\left(z_{1}\right)-\delta_{X}\left(z_{2}\right)\right| \leq\left|z_{1}-z_{2}\right|$, the following assertion can be verified in the same way as in the case of the $j$-metric [4, Thm 7.47, p. 147], [12, p. 36]. 
Lemma 4.2. The function $\hat{j}_{X}: X \times X \rightarrow[0, \infty)$ satisfies the triangle inequality. Moreover, $1 \leq \hat{j}_{X}(x, y) / j_{X}(x, y) \leq 2$ for all $x, y \in X$.

In view of Lemma 4.2, $\hat{j}_{X}$ is a distance on $X$ which is comparable with the $j$-metric $j_{X}$. By Lemma 4.1, for $z_{1}, z_{2} \in \partial E_{j}$ with $1<j<n, \hat{\delta}_{X}(z)=\left|z-a_{j}\right|=\rho_{j}$ and thus $j_{X}\left(z_{1}, z_{2}\right)=\log \left(1+\left|z_{1}-z_{2}\right| / \rho_{j}\right)$. Similarly, we have $j_{X}\left(z_{1}, z_{2}\right)=\log \left(1+2\left|z_{1}-z_{2}\right| / \rho_{j}\right)$ for $z_{1}, z_{2} \in \partial E_{j}$ with $j=1, n$. If we take $f_{j}(t)=\log (1+2 \sin (t / 2))$ for $1<j<n$ and $f_{j}(t)=\log (1+4 \sin (t / 2))$ for $j=1, n$ and $S_{j}=1$ for $1 \leq j \leq n$ in the above construction, we obtain the relation $D_{j}\left(z_{1}, z_{2}\right)=\hat{j}_{X}\left(z_{1}, z_{2}\right)$ for $z_{1}, z_{2} \in \partial E_{j}, 1 \leq j \leq n$. Since $\hat{j}\left(z_{1}, z_{2}\right)$ is Lipschitz equivalent to the euclidean distance $\left|z_{1}-z_{2}\right|$ on $W$, the next result follows from Theorem 3.4 .

Theorem 4.3. Suppose that $A$ is a subset of $\widehat{\mathbb{C}}$ consisting of $n \geq 3$ points with $0, \infty \in A$ and $X=\widehat{\mathbb{C}} \backslash A$. The distance $d_{X}$ constructed above with $\mu\left(z_{1}, z_{2}\right)=\hat{j}_{X}\left(z_{1}, z_{2}\right)$ satisfies the inequalities

$$
B_{1} d_{X}\left(z_{1}, z_{2}\right) \leq h_{X}\left(z_{1}, z_{2}\right) \leq B_{2} d_{X}\left(z_{1}, z_{2}\right), \quad z_{1}, z_{2} \in X
$$

where $B_{1}$ and $B_{2}$ are positive constants depending on $X$.

Similarly, we can modify the definition of the quasihyperbolic distance by setting

$$
\hat{q}_{X}\left(z_{1}, z_{2}\right)=\inf _{\gamma} \int_{\gamma} \frac{|d w|}{\hat{\delta}_{X}(z)},
$$

where the infimum is taken over all the rectifiable curves $\gamma$ joining $z_{1}$ and $z_{2}$ in $X$. In the proof of (2.1), Martin and Osgood indeed showed that a quasihyperbolic geodesic in $\mathbb{C}^{*}$ is an arc of a logarithmic spiral about the origin. In particular, the punctured disk $0<|z|<r$ is quasihyperbolically convex. Since $1 / \delta_{X}(z) \leq 1 / \hat{\delta}_{X}(z)$, Lemma 4.1 implies that $E_{j}^{*}$ is convex with respect to the metric $\hat{q}_{X}$ and the following representation is valid:

$$
\hat{q}_{X}\left(z_{1}, z_{2}\right)= \begin{cases}q_{X}\left(z_{1}, z_{2}\right)=q\left(z_{1}-a_{j}, z_{2}-a_{j}\right), & z_{1}, z_{2} \in E_{j}^{*}, 1<j<n, \\ q\left(z_{1}, z_{2}\right) / 2, & z_{1}, z_{2} \in E_{j}^{*}, j=1, n .\end{cases}
$$

Here, $q\left(z_{1}, z_{2}\right)$ is given in (2.1). In particular, we obtain

$$
\hat{q}_{X}\left(z_{1}, z_{2}\right)= \begin{cases}\theta, & z_{1}, z_{2} \in \partial E_{j}^{*}, 1<j<n \\ \theta / 2, & z_{1}, z_{2} \in \partial E_{j}^{*}, j=1, n\end{cases}
$$

where $\theta=\left|\arg \left(\left(z_{2}-a_{j}\right) /\left(z_{1}-a_{j}\right)\right)\right| \in[0, \pi]$ for $1 \leq j<n$ and $\theta=\left|\arg \left(z_{2} / z_{1}\right)\right| \in[0, \pi]$ for $j=n$. Therefore, in the construction of $d_{X}$ in the previous section, we can choose $f_{j}(t)=(2 / \pi) t$ for $j=1, \ldots, n, S_{j}=\pi / 2$ for $1<j<n$ and $S_{j}=\pi / 4$ for $j=1, n$.

Theorem 4.4. Let $X=\widehat{\mathbb{C}} \backslash A$ be an n-times punctured sphere with $X \subset \mathbb{C}_{0,1}$. Then the distance $d_{X}\left(z_{1}, z_{2}\right)$ on $X$ constructed above with $\mu\left(z_{1}, z_{2}\right)=\hat{q}_{X}\left(z_{1}, z_{2}\right)$ is comparable with the hyperbolic metric on $X$ :

$$
B_{1} d_{X}\left(z_{1}, z_{2}\right) \leq h_{X}\left(z_{1}, z_{2}\right) \leq B_{2} d_{X}\left(z_{1}, z_{2}\right), \quad z_{1}, z_{2} \in X
$$

where $B_{1}$ and $B_{2}$ are positive constants depending only on $Q(A)$. 
In Corollary 3.5 and Theorem 4.3, the constants $B_{1}$ and $B_{2}$ may depend on individual $X$ (more precisely, on $\rho_{\min }$ and $\rho_{\max }=\rho_{n}$ ). In view of Proposition 4.7, we may choose $B_{1}$ and $B_{2}$ as a function of $\widehat{Q}(A)$. The merit of this theorem is that the constants depend on $Q(A)$ only.

We will need general estimates of the hyperbolic metric. Following Beardon and Pommerenke [9], we define the technical but useful quantity

$$
\beta_{\Omega}(z)=\inf \left\{|\log | \frac{z-a}{b-a}||: a, b \in \partial \Omega,|z-a|=\delta_{\Omega}(z)\right\}
$$

for $z \in \Omega \subset \mathbb{C}$. Note that $\beta_{\Omega}(z)=\beta_{f(\Omega)}(f(z))$ for an affine automorphism $f(z)=$ $k z+l, k \neq 0$, of $\mathbb{C}$. The following result describes nicely the behaviour of the hyperbolic metric.

Lemma 4.5 (Beardon-Pommerenke [9]).

$$
C_{2} \leq \lambda_{\Omega}(z) \delta_{\Omega}(z)\left(C_{1}+\beta_{\Omega}(z)\right) \leq C_{3},
$$

where $C_{1}, C_{2}, C_{3}$ are positive absolute constants.

Indeed, Beardon and Pommerenke [9] gave $C_{1}=4+\log (3+2 \sqrt{2}), C_{2}=1 / 2 \sqrt{2}, C_{3}=$ $C_{1}+\pi / 4$. The proof of Theorem 4.4 will be based on the following lemma.

Lemma 4.6. Let $A$ be a finite subset of $\widehat{\mathbb{C}}$ with $n=\operatorname{card}(A) \geq 3$ and $\infty \in A$. Then $X=\widehat{\mathbb{C}} \backslash A$ satisfies the inequality

$$
\beta_{X}(z) \leq \max \left\{\frac{1}{2} Q(A)+c, \log (1+2 e)\right\}, \quad z \in W
$$

where $W$ is as in Theorem 3.4 and $c=\frac{1}{2} \log [1+1 /(4 e(1+e))] \approx 0.012217$.

Proof. Put $Q=Q(A)$. We may assume that $0 \in A$ because of the affine invariance of the quantities involved. As before, we enumerate $A$ as $a_{1}, \ldots, a_{n}$ so that $a_{n}=\infty$ (but we do not assume $a_{1}=0$ at this time). On the contrary, suppose that $\beta_{0}:=\beta_{X}\left(z_{0}\right)>$ $\max \{Q / 2+c, \log (1+2 e)\}$ for some point $z_{0} \in W$. Note here that $\log (1+2 e) \approx 1.86199$. Then $\delta_{0}:=\delta_{X}\left(z_{0}\right)=\left|z_{0}-a\right|$ for some $a \in A$. We assume $a=a_{1}$. By the definition of $\beta_{X}\left(z_{0}\right)$, the set $A$ decomposes into the two sets $A_{1}=\left\{a_{j}:\left|a_{j}-a_{1}\right| \leq e^{-\beta_{0}} \delta_{0}\right\}$ and $A_{2}=\left\{a_{j}:\left|a_{j}-a_{1}\right| \geq e^{\beta_{0}} \delta_{0}\right\}$. Suppose that $\operatorname{card}\left(A_{1}\right) \geq 2$ and $\operatorname{card}\left(\overline{A_{2}}\right) \geq 2$. Then $A=A_{1} \sqcup A_{2}$ and thus

$$
\left|\operatorname{cr}\left(a_{1}, a_{j}, a_{k}, a_{n}\right)\right|=\frac{\left|a_{k}-a_{1}\right|}{\left|a_{j}-a_{1}\right|} \geq e^{2 \beta_{0}}
$$

for $a_{j} \in A_{1} \backslash\left\{a_{1}\right\}, a_{k} \in A_{2} \backslash\left\{a_{n}\right\}$. In view of (2.3) , we have thus $P\left(A_{1}, A_{2}\right) \geq \log \left(e^{2 \beta_{0}}-1\right)=$ $2 \beta_{0}-f\left(2 \beta_{0}\right)$, where $f(x)=-\log \left(1-e^{-x}\right)$. Since $f(x)$ is decreasing in $0<x<+\infty$, we observe that $f\left(2 \beta_{0}\right)<f(2 \log (1+2 e))=\log \frac{1+4 e+4 e^{2}}{4 e+4 e^{2}}=2 c$. Hence, $Q(A) \geq P\left(A_{1}, A_{2}\right)>$ $2 \beta_{0}-2 c$, which is a contradiction.

Therefore, we should have either

(i) $\left|a_{j}-a_{1}\right| \leq e^{-\beta_{0}} \delta_{0}$ for all $1<j<n$, or else

(ii) $\left|a_{j}-a_{1}\right| \geq e^{\beta_{0}} \delta_{0}$ for all $1<j<n$. 
In the case (ii), we have $\left|z_{0}-a_{1}\right|=\delta_{0} \leq e^{-\beta_{0}} \tilde{\rho}_{1}<\rho_{1}$ because $\beta_{0}>1$. Therefore, $z_{0} \in E_{1}$, which violates the assumption $z_{0} \in W$. Thus, the case (ii) does not occur. Set $M=\max _{1<j<n}\left|a_{j}-a_{1}\right|$. Then we have $e^{\beta_{0}} M \leq \delta_{0}=\left|z_{0}-a_{1}\right|$. If $a_{1}=0$, then $M=\tilde{\rho}_{n}$ and $\rho_{n}<e^{\beta_{0}} \tilde{\rho}_{n} \leq\left|z_{0}\right|$, which implies $z_{0} \in E_{n}$, a contradiction. If $a_{1} \neq 0$, then $a_{k}=0$ for some $1<k<n$. In particular, $\left|a_{1}\right|=\left|a_{k}-a_{1}\right| \leq M$. Therefore, $e^{\beta_{0}} M \leq\left|z_{0}\right|+\left|a_{1}\right| \leq\left|z_{0}\right|+M$, which yields $M \leq\left|z_{0}\right| /\left(e^{\beta_{0}}-1\right)$. On the other hand, $\left|a_{j}\right| \leq\left|a_{j}-a_{1}\right|+\left|a_{1}\right| \leq 2 M$ for $1<j<n$. Therefore, $\rho_{n} / e=\tilde{\rho}_{n} \leq 2 M \leq 2\left|z_{0}\right| /\left(e^{\beta_{0}}-1\right)<\left|z_{0}\right| / e$, where we used the assumption $\beta_{0}>\log (1+2 e)$. Thus, we obtain $\left|z_{0}\right|>\rho_{n} e$, which implies $z_{0} \in E_{n}$. This contradicts again the assumption $z_{0} \in W$. Hence, the conclusion follows.

The next result gives some inequalities for the numbers $\rho_{j}$ in terms of $\widehat{Q}(A)$.

Proposition 4.7. Let $A$ be a finite subset of $\widehat{\mathbb{C}}$ with $0,1, \infty \in A$. Then,

$$
\begin{gathered}
\frac{1}{2} \exp \left(\frac{1}{2} \widehat{Q}(A)+2\right) \leq \frac{\rho_{n}}{\rho_{\min }} \leq 2 \exp (\widehat{Q}(A)+2) \\
\frac{1}{2} \exp (-\widehat{Q}(A)-1) \leq \rho_{\min } \leq e^{-1} \text { and } e \leq \rho_{n} \leq \exp (\widehat{Q}(A)+1) .
\end{gathered}
$$

Proof. Recall that $A=\left\{a_{1}, \ldots, a_{n}\right\}$ with $0,1 \in A$ and $a_{n}=\infty$. We also recall that $\tilde{\rho}_{j}=e \rho_{j}=\min _{k \neq j}\left|a_{k}-a_{j}\right|$ for $j=1, \ldots, n-1$ and $\tilde{\rho}_{n}=\rho_{n} / e=\max _{j \neq n}\left|a_{j}\right| \geq 1$. We make a preliminary observation. For a triple $a_{j}, a_{k}, a_{l}$ of distinct finite points in $A$, we have

$$
\frac{\left|a_{k}-a_{j}\right|}{\left|a_{l}-a_{j}\right|} \leq \frac{2 \tilde{\rho}_{n}}{\tilde{\rho}_{j}} \leq \frac{2 \rho_{n}}{e^{2} \rho_{\min }}
$$

Put $Q=\widehat{Q}(A)$. By the definition of $\widehat{Q}(A)$, there is a quadruple $a_{j}, a_{k}, a_{l}, a_{m}$ such that $e^{Q}=\left|\operatorname{cr}\left(a_{j}, a_{k}, a_{l}, a_{m}\right)\right|$. If none of them is $\infty$, we estimate $Q$ by

$$
e^{Q}=\frac{\left|a_{l}-a_{j}\right|}{\left|a_{k}-a_{j}\right|} \cdot \frac{\left|a_{k}-a_{m}\right|}{\left|a_{l}-a_{m}\right|} \leq\left(\frac{2 \rho_{n}}{e^{2} \rho_{\min }}\right)^{2},
$$

which implies the first inequality. Otherwise, we have the better estimate $e^{Q} \leq 2 a_{n} / e^{2} \rho_{\min }$.

Next we show the second inequality. We may assume that $e \rho_{\min }=\left|a_{2}-a_{1}\right|$ and $\rho_{n} / e=\left|a_{3}\right|$. Then,

$$
\frac{\rho_{n}}{e^{2} \rho_{\min }}=\frac{\left|a_{3}\right|}{\left|a_{2}-a_{1}\right|} \leq\left|\operatorname{cr}\left(a_{1}, a_{2}, a_{3}, \infty\right)\right|+\left|\operatorname{cr}\left(a_{1}, a_{2}, 0, \infty\right)\right| \leq 2 e^{Q},
$$

which leads to the second inequality. Since $\rho_{n} \geq e$, the second inequality yields the third one. Finally, we have

$$
e^{-1} \rho_{n}=\left|a_{3}\right|=\left|\operatorname{cr}\left(a_{3}, \infty, 0,1\right)\right| \leq e^{Q}
$$

which completes the proof.

Proof of Theorem 4.4. We will show that $K_{1} h_{X}\left(z_{1}, z_{2}\right) \leq \hat{q}_{X}\left(z_{1}, z_{2}\right) \leq K_{2} h_{X}\left(z_{1}, z_{2}\right)$ for $z_{1}, z_{2} \in W$, where $W$ is as in Theorem 3.4 and $K_{1}$ and $K_{2}$ are constants depending only on $Q(A)$. Then Theorem 3.4 will provide the required assertion. Since $\lambda_{X}(z) \leq 1 / \delta_{X}(z) \leq$ $1 / \hat{\delta}_{X}(z)$, it is clear that the inequality $h_{X}\left(z_{1}, z_{2}\right) \leq \hat{q}_{X}\left(z_{1}, z_{2}\right)$ holds for $z_{1}, z_{2} \in X$. In order 
to show the other inequality, we take a shortest hyperbolic geodesic $\alpha$ joining $z_{1}$ and $z_{2}$ in $X$ for $z_{1}, z_{2} \in W$. By Lemmas 4.5 and 4.6, we have the estimate

$$
\lambda_{X}(z) \geq \frac{K}{\delta_{X}(z)}, \quad z \in W
$$

where $K=K(Q(A))$ is a constant depending only on $Q(A)$. More concretely, one can choose

$$
K=\frac{C_{3}}{C_{1}+\max \{Q(A) / 2+c, \log (1+2 e)\}} .
$$

Hence,

$$
h_{X}\left(z_{1}, z_{2}\right)=\int_{\alpha} \lambda(z)|d z| \geq K \int_{\alpha} \frac{|d z|}{\hat{\delta}_{X}(z)} \geq K \hat{q}_{X}\left(z_{1}, z_{2}\right)
$$

if $\alpha$ is contained in $W$. Otherwise, we take a connected component $\alpha_{1}$ of $\alpha \cap E_{j}$. Then the endpoints $\zeta_{1}, \zeta_{2}$ of $\alpha_{1}$ lie on the circle $\partial E_{j}$. Choose $a_{k}$ so that $\tilde{\rho}_{j}=\left|a_{k}-a_{j}\right|\left(\tilde{\rho}_{n}=\left|a_{k}\right|\right.$ when $j=n)$. Then the function $w=g(z)=\left(z-a_{j}\right) /\left(a_{k}-a_{j}\right)$ maps $X$ into $\mathbb{C}_{0,1}$ (let $g(z)=a_{k} / z$ when $\left.j=n\right)$. By Lemma 3.1 with $f=$ id and (4.1), we obtain

$$
\begin{aligned}
h_{X}\left(\zeta_{1}, \zeta_{2}\right) & \geq h_{\mathbb{C}_{0,1}}\left(g\left(\zeta_{1}\right), g\left(\zeta_{2}\right)\right) \geq \frac{1}{M_{0}} D^{\mathrm{id}}\left(g\left(\zeta_{1}\right), g\left(\zeta_{2}\right)\right)=\frac{\theta}{M_{0}} \\
& =\frac{1}{M_{0}} q\left(g\left(\zeta_{1}\right), g\left(\zeta_{2}\right)\right)=\frac{1}{M_{0}} q_{X}\left(\zeta_{1}, \zeta_{2}\right) \geq \frac{1}{2 M_{0}} \hat{q}_{X}\left(\zeta_{1}, \zeta_{2}\right),
\end{aligned}
$$

where $\theta=\left|\arg \left(\zeta_{1}-a_{j}\right) /\left(\zeta_{2}-a_{j}\right)\right| \in[0, \pi]$. By using the triangle inequality for $\hat{q}$, we now obtain $\hat{q}_{X}\left(z_{1}, z_{2}\right) \leq K_{2} h_{X}\left(z_{1}, z_{2}\right)$ with $K_{2}=\max \left\{1 / K, 2 M_{0}\right\}$.

\section{REFERENCES}

1. C. Adams, Maximal cusps, collars, and systoles in hyperbolic surfaces, Indiana Univ. Math. J. 47 (1998), 419-437.

2. S. Agard, Distortion theorems for quasiconformal mappings, Ann. Acad. Sci. Fenn. A I Math. 413 (1968), 1-12.

3. L. V. Ahlfors, Conformal Invariants, McGraw-Hill, New York, 1973.

4. G. D. Anderson, M. K. Vamanamurthy, and M. K. Vuorinen, Conformal Invariants, Inequalities, and Quasiconformal Maps, Wiley-Interscience, 1997.

5. F. G. Avkhadiev and K.-J. Wirths, Schwarz-Pick Type Inequalities, Frontiers in Mathematics, Birkhäuser Verlag, Basel, 2009.

6. A. F. Beardon, The geometry of discrete groups, Graduate Texts in Mathematics, vol. 91, SpringerVerlag, New York, 1983.

7. _ The hyperbolic metric of a rectangle, Ann. Acad. Sci. Fenn. Math. 26 (2001), 401-407.

8. $\quad$ The hyperbolic metric of a rectangle II, Ann. Acad. Sci. Fenn. Math. 28 (2003), 143-152.

9. A. F. Beardon and Ch. Pommerenke, The Poincaré metric of plane domains, J. London Math. Soc. (2) 18 (1978), 475-483.

10. D. Betsakos, Estimation of the hyperbolic metric by using the punctured plane, Math. Z. 259 (2008), 187-196.

11. F. P. Gardiner and N. Lakic, Comparing Poincaré densities, Ann. of Math. 154 (2001), 245-267.

12. F. W. Gehring and K. Hag, The Ubiquitous Quasidisk, Amer. Math. Soc., Providence, RI, 2012.

13. V. Gol'dshtein and A. Ukhlov, On the first eigenvalue of free vibrating membranes in conformal regular domains, Arch. Ration. Mech. Anal. 221 (2016), 893-915.

14. N. Halpern, A proof of the collar lemma, Bull. London Math. Soc. 13 (1981), 141-144.

15. W. K. Hayman, Subharmonic Functions. Vol. 2, Academic Press, London, 1989. 
16. J. Heinonen, Lectures on Analysis on Metric Spaces, Springer-Verlag, New York, 2001.

17. J. A. Hempel, The Poincaré metric on the twice punctured plane and the theorems of Landau and Schottky, J. London Math. Soc. (2) 20 (1979), 435-445.

18. D. A. Herron, W. Ma, and D. Minda, Möbius invariant metrics bilipschitz equivalent to the hyperbolic metric, Conform. Geom. Dyn. 12 (2008), 67-96.

19. P. Järvi and M. Vuorinen, Uniformly perfect sets and quasiregular mappings, J. London Math. Soc. 54 (1996), 515-529.

20. L. Keen and N. Lakic, Hyperbolic Geometry from a Local Viewpoint, Cambridge University Press, Cambridge, 2007.

21. S.-A. Kim, J. Ma, and W. Ma, Estimates of the hyperbolic metric on the twice punctured plane. Ann. Acad. Sci. Fenn. Math. 40 (2015), no. 2, 875-887.

22. D. Kraus and O. Roth, Conformal metrics, Topics in Modern Function Theory, Ramanujan Math. Soc. Lect. Notes Ser. 19, Ramanujan Math. Soc, Mysore, 2013, pp. 41-83.

23. _ Sharp lower bounds for the hyperbolic metric of the complement of a closed subset of the unit circle and theorems of Schwarz-Pick-, Schottky- and Landau-type for analytic functions, Constr. Approx. 43 (2016), 47-69.

24. W. Ma and D. Minda, The hyperbolic metric and uniformly perfect regions, J. Anal. 20 (2012), 59-70.

25. G. J. Martin and B. G. Osgood, The quasihyperbolic metric and associated estimates on the hyperbolic metric, J. Analyse Math. 47 (1986), 37-53.

26. R. Nevanlinna, Eindeutige Analytische Funktionen, Springer-Verlag, New York-Berlin, 1953, English translation: Analytic Functions, Springer-Verlag, 1970.

27. S.-L. Qiu, Singular values, quasiconformal maps and the schottky upper bound, Sci. China Ser. A 41 (1998), 1241-1247.

28. S. Rickman, Quasiregular mappings and metrics on the n-sphere with punctures, Comment. Math. Helv. 59 (1984), 134-148.

29. P. Schmutz, Congruence subgroups and maximal Riemann surfaces, J. Geom. Anal. 4 (1994), 207-218.

30. A. Yu. Solynin and M. Vuorinen, Estimates for the hyperbolic metric of the punctured plane and applications, Israel J. Math. 124 (2001), 29-60.

31. T. Sugawa, Various domain constants related to uniform perfectness, Complex Variables Theory Appl. 36 (1998), 311-345.

32. Uniform perfectness of the limit sets of Kleinian groups, Trans. Amer. Math. Soc. 353 (2001), $3603-3615$.

33. A conformally invariant metric on Riemann surfaces associated with integrable holomorphic quadratic differentials, Math. Z. 266 (2010), 645-664.

34. _ Modulus techniques in geometric function theory, Journal of Analysis 18 (2010), 373-397, The Proceedings of the ICM2010 Satellite Conference: International Workshop on Harmonic and Quasiconformal Mappings (HQM2010) August 09-17, 2010.

35. T. Sugawa and M. Vuorinen, Some inequalities for the Poincaré metric of plane domains, Math. Z. 250 (2005), 885-906.

36. T. Sugawa and T. Zhang, Construction of nearly hyperbolic distance on punctured spheres, preprint $\mathbf{n} / \mathbf{a}(2017), 12$ pages.

37. J. Väisälä, Free quasiconformality in Banach spaces I, Ann. Acad. Sci. Fenn. Math. 15 (1990), 355379 .

38. _ Q Quasihyperbolic geometry of planar domains, Ann. Acad. Sci. Fenn. Math. 34 (2009), 447473.

39. A. Yamada, On Marden's universal constant of Fuchsian groups. II, J. Analyse Math. 41 (1982), $234-248$. 
Graduate School of Information Sciences, Tohoku University, Aoba-ku, Sendai 9808579, JAPAN

E-mail address: sugawa@math.is.tohoku.ac.jp

Department of Mathematics and Statistics, University of Turku, Fi-20014 Turku, FinLAND

E-mail address: vuorinen@utu.fi

Department of Mathematics, Soochow University, No.1 Shizi Street, Suzhou 215006 , CHINA

E-mail address: trzhang@suda.edu.cn 